Negative correlations were noticed between serum IgA levels and lactosis levels.

Conclusions Serum levels of immunoglobulins in infants (both IgA and IgG) seems to be more related to human milk IgG and lactosis levels rather than human milk $\operatorname{IgA}$ and their specific chemokines.

\section{INVESTIGATING THE EFFECT OF BLACK TEA CONSUMPTION DURING PREGNANCY ON THE OXIDANT/ ANTIOXIDANT STATUS OF BREAST MILK}

doi:10.1136/archdischild-2012-302724.0496

${ }^{1} \mathrm{SM}$ Kayıran, ${ }^{2} \mathrm{DA}$ Ince, ${ }^{3} \mathrm{D}$ Aldemir, ${ }^{4 \mathrm{~B}}$ Gürakan. 'Deapartment of Pediatrics, American Hospital, Istanbul; ' 2 epartment of Pediatrics, Division Neonatology; ${ }^{3}$ Department of Biochemistry, Baskent University Hospital, Ankara; ${ }^{4}$ Department of Pediatrics, Division of Neonatology, American Hospital, Istanbul, Turkey

Background and Aims Black tea is associated with antioxidant properties. The objective of this study was to investigate the effect of the amount of black tea consumption during pregnancy on the oxidant/antioxidant status of breast milk.

Methods Breast milk was obtained from 30 mothers and analysed for lipid peroxidation based on levels of malondialdehyde (MDA), and levels of reduced glutathione (GSH). In a survey completed by all participants, daily black tea consumption during the pregnancy was reported.

Results No correlation was found between the amount of black tea consumed and levels of MDA ( $p=0.401)$ and/or GSH detected $(p=0.473)$. The results of this study indicate that consumption of varying amounts of black tea do not affect the oxidant/antioxidant status of breast milk.

Conclusions We conclude that this insensitivity of breast milk to antioxidant contributions by black tea reflects the capacity for breast milk to be resistant to the myriad of factors that otherwise affect a pregnant woman.

\section{EBF IN NICU AND NORMAL NURSERY BABIES IN IRAN}

doi:10.1136/archdischild-2012-302724.0497

R Saeedi, S Raouf YazdiNejad, S Rahmani. Mashhad University of Medical Sciences, Mashhad, Iran

Introduction Exclusive breastfeeding (EBF) that means "no supplemental liquids or solid foods other than medications or vitamins" is what recommended by WHO for the first 6 months of life to decrease the mortality and morbidity in infants. Statistics indicate, however, that initiation and maintenance of EBF are becoming low in our country.

Method To identify the reasons of unsuccessful EBF in 150 motherinfant pairs hospitalized in two different wards (NICU and normal nursery) in two public hospitals in 2008 in Mashhad, we conducted a prospective study which contains 3 stages. At first mothers were interviewed in hospital at the time of discharge and completed 2 telephone interviews at 3 and 6 months postpartum. Finally 129 mothers remained in the study.

Results We found that $65.1 \%$ of mothers whose infants had been hospitalized in NICU initiated EBF, \%51.6 continued EBF until 3 months and $40.3 \%$ completed EBF until 6 months postpartum. These percents were respectively $85.1 \%, 70.1 \%$ and $49.3 \%$ for normal nursery infants' mothers. Also we found that hospitalization in NICU, lower birth weight and gestational age, longer duration of hospitalization and NPO duration and having more than two infants in one delivery have negative effects on being successful in EBF

Conclusion Prevention and timely resolution of breastfeeding problems among NICU infants' mothers is what we recommend based on this study results. Better quality lactation training for both mothers and their husbands may be useful too.

\section{AWARENESS, PRACTICES AND BENEFITS OF BREAST FEEDING IN ISRA UNIVERSITY HOSPITAL, PAKISTAN}

doi:10.1136/archdischild-2012-302724.0498

A Baig. Community Medicine, Isra University Pakistan, Hyderabad, Pakistan

Background Several studies regarding Breastfeeding have been done in Pakistan and around the world. We have also done such a study in Isra University Hospital. Breast feeding is perfectly suited to nourish infants. It is truly said that what is good for babies is good for mother.

Our objectives were to determine knowledge about optimum breast feeding practices, benefits of breast feeding and pre-lacteal feeds, to evaluate the awareness of breast feeding through demographic parameters and to determine the causes of failure of breast feeding among mothers.

Methodology Questionnaire based hospital survey was conducted among 150 mothers, based on objectives which depended on certain variables: demographic variables like age, education, socio economic status, occupation, address. It also had questions about duration of marriage, number of kids. Study design was Cross Sectional Study and was done using SPSS.

Results Breast feeding was initiated by $89.33 \%$ (134) of mothers. $10.67 \%$ (16) of mothers, who failed to breastfeed their babies, had mainly the reason of milk not being produced (50\%). Later we specifically asked the breastfeeding mothers certain questions. It was revealed that they were breast feeding their babies because breast milk is the best/balanced diet. $84 \%$ recommended breastfeeding for today's mothers.

Conclusion According to our study Illiterate females and house wives were breast feeding more. Economically deprived mothers tended to breastfeed their babies for longer period as compared to economically well-off or educated mothers. We came to the conclusion that awareness programs regarding correct breastfeeding practices be conducted.

\section{EXCLUSIVE BREAST-FEEDING AND RELATED FACTORS IN INFANTS}

doi:10.1136/archdischild-2012-302724.0499

${ }^{1} \mathrm{~A}$ Azarfar, ${ }^{2} \mathrm{Y}$ Ravanshad, ${ }^{3 Z}$ Khamnian. ${ }^{1}$ Pediatric, Mashhad University of Medical Sciences, Mashhad; ${ }^{2}$ Comunity Medicine; ${ }^{3}$ Assistant Community Medicine, Tabriz University of Medical Sciences, Tabriz, Iran

Introduction Breast feeding method that provides physical, psychological and spiritual needs, in first 6 months of birth. Exclusive breast feeding up to 6 months, is a major factor in health financing in developing countries.

Method It is a cross sectional study in breastfeeding mothers came to public health centers in one year at Tabriz in this study selected 150 mother with bellow 2 years old baby with simple random sampling we used a valid questioner we used X2 test and SPSS16 software for analyze of data.

Results and findings In this study frequency of exclusive breastfeeding up to 6 months were 56/4\% (49/3-63/4: C195\%) with rate in boys $57 / 2 \%(49 / 9-64 / 4$ : CI95\%)and in girls were $55 / 7 \%(62 / 6$ 48/7: CI95\%) and 91/6\% (94/7-88/7: CI95\%) were children who had breast feeding immediately after delivery, of which $41 / 3$ percent had continued feeding up to six months. study showed a direct relationship between longitude of breast feeding and educational study before delivery and a indirect relationship with maternal age. Maternal age, maternal knowledge and advice of relevant about use of formula were meaningful factors in the multiple regression model Most of the mothers (85.4\%) were in middle socio-economic group. 
in Knowledge of mothers about breast feeding, $42.5 \%$ had good knowledge, $56.5 \%$ had average knowledge.

Conclusion Raise awareness of breast-feeding mothers than exclusively breastfeeding, were the important health priorities in the health of children.

feeding, breastfeeding, attitudes, mothers, infants

\section{DILATATIVE CARDIOMYOPATHY - CASE REPORT}

doi:10.1136/archdischild-2012-302724.0500

MV Sofranac. Paediatric, Health Centre Danilovgrad, Danilovgrad, Montenegro

Background and Aims Dilatative cardiomyopathy/DC/is a myocardium disease characterized by increased dimension of heart cavites and general weakening of the systolic function/the most frequently of the left ventricle/, with emersion ofsymptoms and signs of a cardiac insufficiency. Etiologically, there are family and genetic factors. Manifestations of the disease at infants are feeding problems, difficult and accelerated breathing and excessive sweating. Older children complain of fatigue, difficult breathing and hacking. It can olso be asymptomatic and discovered at occasional medical examination with an x-ray finding of cardiomegaly or electrocardiogram changes, or with an appearance of an unspecific symptom, as was he case here.

Methods Data analysis of the case history of the patient with DC. Anamnestic data, laboratory analysis and data through clinical diagnostic procedures of reference institutions are used as work methods. Purpose is to show the patient with a diagnosed DC with an initial unspecific symptom.

Results The work show a boy old 14 with dyspnea as the only symptom. Forth child of the fifth normal pregnancy/death of the two-month old sister caused by a heart condition of unknown etiology/. Slowed development as infant, frequent respiratory infections. A boy adipose, tachypneic, dyspneic. Sistolic murmur at apex. TA100/60mmHg, CP100/min, sO290\%.X-ray:cardiomegaly. In competent institution diagnosis is confirmed.

Conclusions Initial clinical presentation of the disease at patients with DC can be characterized by unspecific respiratory disturbances, as was the case with this patient. Echocardiography remains a sovereign method in establishing the diagnosis. In the future, endomyocardial biopsy is expected to offer decisive data regarding the etiopathogenesis of this condition which would enable a timely causal medication therapy and avoid surgical therapy.

\section{THE INFLUENCE OF BETA - BLOCKER (BISOPROLOL) ON HEART RATE VARIABILITY IN CHILDREN WITH MITRAL VALVE PROLAPSE}

doi:10.1136/archdischild-2012-302724.0501

'L Romanciuc, ${ }^{2,3} \mathrm{~N}$ Revenco. 'Pediatrics Department, State Medical and Pharmaceutical University 'N. Testemitanu'; 'Pediatrics Department; ${ }^{3}$ Research Institute for Maternal and Child Healthcare, Chisinau, Moldova

The carried out research has included 50 children: I group (bisoprolol)- $60 \%$ children and II group (placebo) - 40.0\%, average age (13.5 \pm 0.60 ) years; doses of bisoprolol (PO): $1.25-2.5 \mathrm{mg} / \mathrm{kg} / \mathrm{dose}$.

ECG monitoring has defined heart rate average maximum and minimum value in the beginning of research and in dynamics a month later. Dynamics in a month of action of a bisoprolol has not changed the minimum values of heart rate in the first group in comparison with placebo ( $p>0.05)$, but has lowered the maximum values of heart rate reductions at children with MVP $(-8.74 ; p<0.01)$ in the first group in comparison with placebo $(0.55 ; p>0.05)$ and average values of heart rate $(-4.70 ; p<0,001)$ in the first group in comparison with placebo $(-0.10 ; p>0.05)$. Statistical parametres HRV (SDNN and PNN 50) defined in the beginning in both groups did not differ considerably $(\mathrm{p}>0.05)$. Dynamics in a month of action bisoprolol on indicators HRV was showed by decrease in value PNN 50 in the first group $(-6.42 ; \mathrm{p}<0,001)$.

Results of research have revealed, that at children with MVP, bisoprolol has considerably reduced heart rate $(-8.9$; $p<0,001)$ in comparison with placebo, without influence on systolic and diastolic blood pressure. Influence bisoprolol on indicators HRV in the first group it was characterised by decrease of statistics PNN 50 characteristic for activity of sympathetic vegetative nervous system $(-6.42 ; \mathrm{p}<0,001)$.

\section{EXPLAINING THE RELATION OF SOCIOECONOMIC STATUS TO CHILDHOOD BLOOD PRESSURE. THE ABCD-STUDY}

doi:10.1136/archdischild-2012-302724.0502

${ }^{1,2}{ }^{2} \mathrm{G}$ van den Berg, ${ }^{2} \mathrm{M}$ van Eijsden, ${ }^{3} \mathrm{TGM}$ Vrijkotte, ${ }^{1} \mathrm{RJBJ}$ Gemke. ${ }^{1}$ Pediatrics, VU University Medical Center; ${ }^{2}$ Epidemiology, Documentation and Health Promotion, Public Health Service of Amsterdam; ${ }^{3}$ Public Health, Academic Medical Center, University of Amsterdam, Amsterdam, The Netherlands

Objective We investigated the association of socioeconomic status to blood pressure and prehypertension in childhood.

Methods In a prospective cohort study (ABCD-study) we obtained blood pressure measurements and information on potential explaining factors, namely birth weight, breastfeeding duration, and body mass index (BMI) in 3067 children of 5-6 year of age.

Results The systolic- and diastolic blood pressures of children from mid-educated women were $1.0 \mathrm{~mm} \mathrm{Hg}$ higher (95\% CI 0.4-1.7) and $0.9 \mathrm{~mm} \mathrm{Hg}$ higher (95\% CI 0.3-1.4), and the blood pressures of children from low-educated women were $2.2 \mathrm{~mm} \mathrm{Hg}$ higher $(95 \% \mathrm{CI}$ 1.4-3.0) and $1.7 \mathrm{~mm} \mathrm{Hg}$ higher (95\% CI 1.1-2.4), compared to children from high-educated women (models controlled for age, gender, height, and ethnicity). Children of mid- or low-educated mothers were also more likely to have prehypertension (>p90;21\% and $27 \%$ ) compared to children of high educated mothers (13\%). In addition, these associations could partly be explained by birth weight, breastfeeding duration, and BMI, but remained significant following adjustment for these variables. Income adequacy was less clearly associated with prehypertension, even after including potential mediators.

Conclusion The socio-economic status related differences in blood pressure seem to emerge from childhood as the results show a higher blood pressure and more prehypertension in children from lower SES. Improving birth weight, breastfeeding duration, and BMI, might help decreasing the socio-economic disparities, but other factors might also play a role.

\section{SEPARATING THE ASSOCIATIONS OF PROGRAMMING AND TRACKING DURING INFANCY WITH CHILDHOOD BLOOD PRESSURE, THE ABCD STUDY}

doi:10.1136/archdischild-2012-302724.0503

${ }^{1} \mathrm{M}$ de Beer, ${ }^{2} \mathrm{M}$ van Eijsden, ${ }^{3} \mathrm{~T}$ Vrijkotte, ${ }^{4} \mathrm{C}$ Fall, ${ }^{4} \mathrm{C}$ Osmond, ${ }^{1} \mathrm{R}$ Gemke. ${ }^{\text {PPediatrics, }}$ VU University Medical Center; ${ }^{2}$ Epidemiology, Documentation and Health Promotion, Municipal Health Service; ${ }^{3}$ Social Medicine, Academic Medical Center, Amsterdam, The Netherlands; ${ }^{4}$ MRC Lifecourse Epidemiology Unit, Southampton General Hospital, Southampton, UK

Background and Aims In a prospective pregnancy cohort study, we investigated the association of early growth with blood pressure at 5-6 years of age.

Methods Our study was based on 2,338 children who were born healthy at $\geq 37$ completed weeks gestation with on average 7 measures of growth (weight and length) from birth to age 12 months. We used conditional weight, a residual of current weight regressed on prior weights, to represent deviations from expected weight gain from 0 to 1,1 to 3,3 to 6 , and 6 to 12 months. The same method 